\title{
CDKL5 deficiency disorder: a pathophysiology of neural maintenance
}

\author{
Peter C. Kind ${ }^{1}$ and Adrian Bird ${ }^{2}$ \\ 'Simons Initiative for the Developing Brain, Centre for Discovery Brain Sciences, and ²Wellcome Centre for Cell Biology, University of Edinburgh, Edinburgh, United Kingdom.
}

\begin{abstract}
Critical periods are developmental time windows in which functional properties of the brain are particularly susceptible to the organism's experience. It was thought that therapeutic strategies for neurodevelopmental disorders (NDDs) required early life intervention for successful treatment, but previous studies in a mouse model of Rett syndrome indicated that this may not be the case, as some genetic disorders result from disruptions of neuromaintenance. In this issue of the JCI, Terzic et al. provide evidence that defective neuromaintenance also underlies CDKL5 deficiency disorder (CDD). The authors used genetic mouse models to examine the role of CDKL5 protein. Notably, when CDKL5 protein was restored in late adolescent CdkI5-deficient animals, CDD behavioral defects were reversed. These results suggest that genetically or pharmacologically restoring CDKL5 may treat CDD after symptom onset.
\end{abstract}

\section{Developmental windows}

Critical periods have been recognized in biological processes that range from embryonic development to cognitive psychology. To neurobiologists, they represent reproducible time windows during development when functional properties of the brain are particularly susceptible to the organism's experience. This concept became prominent due to the seminal findings of Conrad Lorenz, who described imprinting in birds (1), and Hubel and Wiesel, whose research revealed the effects of early deprivation in the visual system (2). Initially, critical periods were invoked to explain instinctive behavior, but have subsequently been associated with more complex animal behaviors, including cognition and language acquisition. While not specifically applied to neurological disorders, it follows from this programmatic view of brain development that the absence of essential genetic components at key developmental stages would make any attempts to correct the resulting neurological defects futile. Despite challenges to the theoretical basis of this conclusion (see Bateson et al., 1979; ref. 3), it became widely accepted that therapeutic strategies for neurodevelopmental disorders (NDDs) could only be successful if initiated very early in an individual's life.

Conventional wisdom regarding NDDs was directly challenged by the work of Guy et al. (4) using a mouse model of Rett syndrome. The researchers demonstrated that the vast majority of phenotypes present in mice lacking MECP2 (the protein missing in Rett syndrome) are reversed in adults by restoring the gene that encoded the missing protein. These studies undermined the notion that MECP2 plays a distinct role during brain development or that brain function is permanently compromised by absence of MeCP2 in early postnatal life. Furthermore, McGraw and colleagues (5) showed that deletion of the Mecp2 gene in adult mice is lethal, despite development

\section{Related Article: https://doi.org/10.1172/JCl143655}

Conflict of interest: The authors have declared that no conflict of interest exists.

having proceeded normally in the presence of the protein. Not only is MeCP2 apparently dispensable for brain development, but its presence is absolutely required in the mature brain. These findings questioned the broader view that disorders whose clinical features present early in life are always neurodevelopmental in the sense that they result from irrevocable changes to the brain that become fixed during abnormal development. In the case of Rett syndrome, the origin of the disorder is better described as a failure of neuromaintenance, as MeCP2 function does not appear to require a privileged period in brain development.

\section{Defective neuromaintenance}

The findings of Terzic et al. (6) in this issue of the JCI provide evidence that CDKL5 deficiency disorder (CDD) should also be attributed to defective neuromaintenance. CDD, which is caused by mutations in the CDKL5 gene, is a severe condition that presents in the first year or two of life and is characterized by severe seizures, intellectual disability, and features of autism. While CDD was initially referred to as atypical Rett syndrome, it is now recognized as a separate disorder with distinct clinical features (7). Terzic and colleagues employed genetic techniques (6) very similar to those adopted previously to examine the role of MeCP2 $(4,5)$. They first demonstrated that adolescent animals (approximately two months of age) with late deletion of the Cdkl5 gene developed behavioral, physiological, and anatomical phenotypes similar to those found in age-matched animals with constitutive $C d k l 5$ deletion. The researchers went on to express the CDKL5 protein in late-adolescent Cdkl5-KO animals and demonstrated that the knockin mice were more similar to WT mice than to Cdkl5-KO animals (6). It follows that CDKL5 is a neuromaintenance protein that is required continuously throughout adult life. Importantly, the defects caused by the absence of CDKL5 were reversed by restoring the protein during late adult life, 
opening up the possibility that genetic and protein replacement studies may ameliorate the disease in older individuals.

The findings of Terzic et al. (6) are undoubtedly encouraging for families of CDD individuals. However, further research is needed to address questions that follow from the current study. Most pertinent, perhaps, is whether similar findings will be seen in female mice, which are the true genetic model of the majority of cases of CDD. Additionally, a key feature of CDD is severe childhood epilepsy, which is not recapitulated in rodent models. To assess reversibility of this symptom, alternative animal models or seizure induction protocols will be required. A potential caveat to the mouse reexpression model used in the study (6) is that a fraction of neurons expressed CDKL5 prior to gene activation. Thus, although the pretreated animals did develop phenotypes similar to those found in constitutive CDKL5-deficient animals, the possibility remains that the few CDKL5-expressing cells could have primed the brain for better recovery in the adult. Use of AAV viral vectors with high transduction rates in mice to deliver a CDKL5 expression construct, as previously shown for MeCP2, could test this possibility (8). Future experiments will also need to recapitulate the reversal experiment in mice older than 8 weeks to determine whether later reexpression or removal of CDKL5 is similarly effective in ameliorating or inducing phenotypes. Finally, for both Rett syndrome and CDD, the reversibility of more complex cognitive functions has yet to be explored, but will help to establish the efficacy of genetic or pharmacological interventions.

\section{Neurodevelopment or neuromaintenance?}

The stage is set for stratification of the numerous monogenic conditions that affect the brain as either neurodevelopmental or neuromaintenance disorders, by assessing phenotypic reversibility in adult animal models. This essential preclinical work has already begun. For example, $U B E 3 A$, which is mutated in Angelman's syndrome, must be expressed during a critical period during development in rodent models (9). Intriguingly, adult restoration of UBE3A in neurons of the prefrontal cortex restored cellular plasticity. Additionally, some phenotypes caused by mutations in the Shank3 or Syngap genes were alleviated by activation of the relevant gene, even in older animals $(10,11)$, although other defects persisted (3).

The concept of critical periods, which originated more than 100 years ago, has evolved with our understanding of brain development. In the 1960s, it became clear that developmental processes are often not confined to a constant and immutable time window, but can expand or contract depending on the environment and experience (reviewed in refs. 12,13). This flexibility suggested the term "sensitive period" rather than "critical period" (reviewed in refs. 12, 13). Furthermore, it is recognized that while development (and its associated sensitive periods) comprises a series of events that occur in a specific sequence, this typical trajectory is not necessarily the only route to a specific developmental end point (12). Potential treatments for genetic disorders that change the neurodevelopmental trajectory will require us to know more than simply when the trait emerges; we will also need to understand how and why. To achieve this, we need to specify the underlying molecular, cellular, and circuit mechanisms as well as identify any mechanistic redundancy. With this knowledge in hand, there is hope that even disorders that qualify as neurodevelopmental will in due course be treatable.

\section{Acknowledgments}

The authors thank colleagues at the Simons Initiative for the Developing Brain for helpful discussions.
Address correspondence to: Peter C. Kind, Simons Initiative for the Developing Brain, Centre for Discovery Brain Sciences, University of Edinburgh, Hugh Robson Building George Square, Edinburgh EH8 9XD, United Kingdom. Phone: 44.131.651.1762; Email: pkind@ed.ac.uk. Or to: Adrian Bird, Wellcome Centre for Cell Biology, Michael Swann Building, University of Edinburgh, Max Born Crescent, Edinburgh EH9 3BF, United Kingdom. Phone: 44.131.650.5670; Email: a.bird@ed.ac.uk.

1. Lorenz K. Der Kumpan in der Umwelt des Vogels. Der Artgenosse als auslösendes Moment sozialer Verhaltensweisen. JOrnithol. 1935;83:137-213.

2. Hubel DH, Wiesel TN. The period of susceptibility to the physiological effects of unilateral eye closure in kittens. J Physiol. 1970;206(2):419-436.

3. Bateson P. How do sensitive periods arise and what are they for? Anim Behav. 1979;27(2):470-486.

4. Guy J, et al. Reversal of neurological defects in a mouse model of Rett syndrome. Science. 2007;315(5815):1143-1147.

5. McGraw CM, et al. Adult neural function requires MeCP2. Science. 2011;333(6039):186.

6. Terzic B, et al. Temporal manipulation of Cdkl5 reveals essential postdevelopmental functions and reversible CDKL5 deficiency disorder-related deficits. J Clin Invest. 2021;131(20):143655.

7. Olson HE, et al. Cyclin-dependent kinase-like 5 deficiency disorder: clinical review. Pediatr Neurol.2019;97:18-25.

8. Tillotson R, et al. Radically truncated MeCP2 rescues Rett syndrome-like neurological defects. Nature. 2017;550(7676):398-401.

9. Mei Y, et al. Adult restoration of Shank3 expression rescues selective autistic-like phenotypes. Nature. 2016;530(7591):481-484.

10. Creson TK, et al. Re-expression of SynGAP protein in adulthood improves translatable measures of brain function and behavior. Elife. 2019;8:e46752.

11. Ozkan ED, et al. Reduced cognition in Syngap1 mutants is caused by isolated damage within developing forebrain excitatory neurons. $\mathrm{Neu}$ ron. 2014;82(6):1317-1333.

12. Cynader M, Mitchell DE. Prolonged sensitivity to monocular deprivation in dark-reared cats. J Neurophysiol. 1980;43(4):1026-1040.

13. Bateson P. Sensitive periods in behavioural development. Arch Dis Child. 1986;58(2):85-86. 